\title{
Validación de un algoritmo para el cálculo de las dimensiones dentarias basado en el método de Bloise
}

\author{
Validation of an algorithm for calculation of tooth dimensions based on \\ the method of Bloise
}

MA. Rospide ${ }^{1}$ DG. Goroso ${ }^{2,3}$

${ }^{1}$ Cátedra de Odontología Legal y Forense. Facultad de Odontología.

Universidad Nacional de Tucumán. San Miguel de

Tucumán. Argentina.

2Instituto de Medicina

Física y Rehabilitación. Hospital Clínicas. Facultad

de Medicina. Universidad de São Paulo. Brasil.

${ }^{3}$ Núcleo de Pesquisas

Tecnológicas.

Universidad de Mogi das

Cruzes. Brasil.

Correspondencia:

Mónica Alejandra Rospide

Facultad de Odontología.

Universidad Nacional de Tucumán

Av. Benjamín Aráoz 800. (4000)

San Miguel de Tucumán. Argentina

E-mail:morospide@hotmail.com

Fecha de recepción: 10.NOV.2014

Fecha de aceptación: 25.MAR.2015

\section{Resumen}

Introducción: Cuando se estudia material óseo procedente de excavaciones arqueológicas o restos aislados es frecuente que las condiciones de conservación sean deficientes, por lo que la reconstrucción antropométrica de los principales rasgos característicos no se puede observar. Este problema es aún mayor en los casos forenses, en los que en muchas ocasiones la identificación debe realizarse únicamente a través de piezas dentarias. Por este motivo, son necesarios métodos alternativos validados que permitan la identificación de un individuo a partir de elementos dentarios.

Objetivo: Validar el algoritmo, basado en el método de Bloise, comparando las dimensiones características de una pieza dentaria de diámetro mesiodistal conocido, tomado como dato inicial para el cálculo, con las dimensiones calculadas de esa misma pieza dentaria. Materiales y métodos: Materiales: se desarrolló un algoritmo basado en el método de Bloise en Excell Versión 14.0. 2010 para calcular el tamaño de piezas dentarias a partir de una única pieza conocida. Las mediciones se realizaron utilizando un calibre digital, clase 2, con precisión de 0,01 mm, y un calibre dentario, clase 2, con precisión de 0,01 mm. Muestra: se realizaron 106 medidas dentarias, provenientes de cráneos y modelos de yeso, todos seleccionados de la Cátedra de Odontología Legal y Forense. Procedimiento experimental: se midió el diámetro medial de una pieza dentaria conocida y se calculó su dimensión usando el algoritmo de cálculo. Análisis de datos: los datos experimentales se compararon con los valores calculados por el algoritmo aplicando el método de Bland-Altman. Para una mayor precisión del algoritmo se determinó un factor de corrección a partir de los coeficientes de regresión lineal de Pearson $(\mathrm{p}<0,05)$. Finalmente, se compararon de nuevo los valores corregidos y los valores experimentales usando el método de Bland-Altman. Resultados: Aplicando el método de Bland-Altman entre los valores experimentales y los calculados sin corrección se observó una dispersión de hasta dos desviaciones estándar. Este problema fue corregido utilizando el factor de corrección determinado por los coeficientes de Pearson, esto es, $\operatorname{Cor}=(\mathrm{Cal}-0,0183) / 0,9601$. Esta corrección permitió disminuir las diferencias entre el valor experimental y el valor calculado por el algoritmo próximo a $\pm 0,08 \mathrm{~mm}$ (menor que una desviación estándar).

Conclusión: El factor de corrección sugerido para el método de Bloise se acerca más a los datos dentarios medidos experimentalmente. A partir de esta corrección fue posible validar el algoritmo basado en el método de Bloise.

Palabras clave: Diámetro dentario. Bland-Altman. Validación de modelos.

\section{Abstract}

Introduction: When you study bone material from archaeological excavations or isolated remains, it is frequent that storage conditions are poor, so the anthropometric reconstruction of the main characteristic features cannot be observed. This problem is even greater in forensic cases in which, on many occasions, the identification must only be performed through tooth parts. For this reason, it is necessary to use validated alternative methods that allow the identification of an individual from dental fragmented remains.

Goal: Validate the algorithm, based on the Bloise's method, by comparing the characteristic dimensions of a tooth in mesiodistal diameter known, taken as initial data for the calculation, with the calculated dimensions of the same tooth.

Materials and methods: Software: an algorithm based on the Bloise's method in friendly Excel Version 14.0. 2010 was developed to calculate the size of the teeth from a single known piece. Instruments: the measurements were performed using a digital caliper, class 2, with $0.01 \mathrm{~mm}$ accuracy and a tooth gauge, class 2, with $0.01 \mathrm{~mm}$ accuracy. Subjects: were carried out 106 measures teeth, skulls and plaster models, were selected from Chair of Legal and Forensic Dentistry. Experimental procedure: a known tooth medial diameter was measured and its dimension was calculated using the algorithm. Analysis of data: the experimental data were compared with the values calculated by the algorithm using the method of Bland-Altman. For greater accuracy of the algorithm a correction factor was determined from the linear regression of Pearson coefficients $(p<0.05)$. Finally, the corrected values and the experimental values were again compared using the method of Bland-Altman.

Results: When the Bland-Altman's method was applied between the experimental and the calculated values without correction, it was observed a dispersion of up to two standard deviations. This problem was corrected using the correction factor determined by the Pearson coefficient, i.e. $\mathrm{Cor}=(\mathrm{Cal}-0.0183) / 0.9601$. This fix allowed to decrease the bias for differences of $\pm 0.08 \mathrm{~mm}$ (less than one standard deviation) between the experimental value and the value obtained from the algorithm.

Conclusion: Correction factors suggested to Bloise's method is closer to the dental data measured experimentally. From this correction, it was possible to validate the virtual simulator based on the Bloise's method.

Key words: Tooth diameter. Bland-Altman. Validation of models. 


\section{Introducción}

En los grandes accidentes en los cuales aparecen cadáveres fragmentados, cortados o con grandes subdivisiones, a veces son pocos los indicios encontrados para realizar la identificación humana, así como también en los restos hallados largo tiempo después de acaecido un suceso de cualquier índole. Desde épocas remotas, los hombres de ciencia se preocuparon por la identificación y la forma de relacionar a los seres humanos con una serie de medidas que sirvieran de patrón para establecer las proporciones orgánicas que configuran su individualidad ${ }^{1}$. Los egipcios encontraron que la talla es diecinueve veces la longitud del dedo medio, confirmado por Blanc; Vitruvio postula su teoría de la proporciones humanas y Pacioli escribe su Tratado de la Divina Proporción. Entre otros, estos estudios dan el concepto de que el ser humano está sujeto a una métrica indiscutida, aunque variada sea su forma de apreciación ${ }^{1}$. Los estudios antropológicos realizados en América, y sobre todo los relacionados con la antropología dental, han sido muy escasos, específicamente en lo que respecta a las variaciones biológicas de poblaciones humanas y en especial a las formas predominantes de las arcadas dentarias del hombre americano ${ }^{2}$. Por otro lado, para los antropólogos los dientes constituyen una de las fuentes más valiosas de evidencia para la comprensión del curso evolutivo de las poblaciones, para el conocimiento de la biología de las poblaciones y para la identificación de un individuo a partir de restos fragmentados ${ }^{3,4}$.

La importancia de la planimetría dentofacial fue señalada por Carrea en el año 1920 en su tesis de doctorado Ensayos odontométricos, en la que afirma "dadme un diente y yo encontrare a la persona"; con ello ilustraba la importancia que los elementos dentales tenían como aporte a la identificación médicolegal ${ }^{5}$. Carrea relacionó las medidas dentarias y su proporcionalidad con el cráneo; él mismo dijo que "Ios dientes humanos guardan relaciones precisas e inconfundibles entre sí. Sus medidas, su forma, son siempre proporcionales al sujeto. El largo, el ancho y el alto de cada diente es proporcional al diente vecino; es proporcional al conjunto que forma la arcada, es proporcional a la cara, a la cabeza y al sujeto en total".

Las medidas odontométricas de mayor objeto de estudio son el diámetro mesiodistal, definido como la distancia entre los puntos interproximales mesial y distal de mayor contorno ${ }^{6,7}$, debido a que estas dimensiones no se ven afectadas por el desgaste ocasionado por la atrición durante la masticación, las propiedades abrasivas de algunos alimentos y hábitos, ni condiciones patológicas como el bruxismo ${ }^{8}$.
El método de Bloise ${ }^{1}$ considera de suma importancia los diámetros dentarios y por medio de estos la obtención de los diagramas de Bonwill, Hawley, Valderrama, Gysi, Carrea, etc., siempre y cuando se disponga de elementos dentarios que hagan posible su trazado. En el trazado de los odontogramas es necesario recurrir a la suma de los diámetros mesiodistales de un incisivo central, lateral y canino para hallar el radio Bonwill o radio cuerda, para proceder al trazado geométrico de la curva dental. La solución que ofrece se basa en el porcentaje mesiodistal, la razón geométrica y un índice con el que se evalúa cada diente ${ }^{9}$. El Dr. Bloise establece magnitudes proporcionales y una métrica craneofacial que es aplicable en ortodoncia, prótesis y odontología legal. La razón geométrica es una constante que permite calcular las medidas de un diente a partir de un fragmento útil. Con este método es posible calcular el diámetro mesiodistal de las piezas dentarias superiores e inferiores, el diámetro longitudinal de la raíz y el espesor coronario. A partir de sus afirmaciones y sus cálculos matemáticos, el diámetro mesiodistal de los elementos dentarios para estimar la estatura fue validado y ajustado por diversos autores ${ }^{10,11}$. Otro estudio utiliza la métrica dentaria para la determinación del sexo ${ }^{12}$. Sin embargo, con posterioridad al estudio realizado por Bloise ${ }^{1}$ no fueron reportados estudios referidos a la relación entre los diámetros dentarios y las medidas faciales.

Asimismo, la formulación de modelos matemáticos y computacionales para su uso en medicina legal proporciona herramientas importantes para la reconstrucción y el reconocimiento físico de una persona.

En este sentido, el presente trabajo se propone desarrollar un algoritmo basado en el método de Bloise ${ }^{1}$ para el caso 1 y 2 de unidades dentarias conocidas, es decir, a partir de un diente inferior conocido o un fragmento útil y a partir de un diente superior conocido o un fragmento útil. El algoritmo será validado comparando las dimensiones características de una única pieza dentaria de diámetro mesiodistal conocido, tomado como dato inicial para el cálculo, con las dimensiones calculadas de esa misma pieza dentaria. Como consecuencia de esta investigación se obtienen los factores de corrección para el método de Bloise ${ }^{1}$.

\section{Materiales y métodos}

\section{Descripción del algoritmo}

El algoritmo para el cálculo de elementos dentarios basado en el método de Bloise ${ }^{1}$ consiste básicamente en un sistema de entrada-salida. La entrada 
es el diámetro mesiodistal coronario, medido en milímetros, de una pieza dentaria conocida superior o inferior: incisivo central, incisivo lateral, canino, primer premolar, segundo premolar, primer molar, segundo molar o tercer molar.

A partir del diámetro mesiodistal de un diente superior, el sistema calcula los diámetros mesiodistales de los restantes dientes superiores, utilizando como valor de referencia los porcentajes de Bloise en función de la hemiarcada ${ }^{1}$ (Tabla 1 ).

Luego se procede al cálculo del radiocuerda (inferior), en función del radio Bondwill (superior) y se calcula la arcada inferior de la misma forma que en el caso de los dientes superiores, utilizando los porcentajes de Bloise para el hemiarco inferior (Tabla 1 ).

En caso de tener una medida útil de diámetro mesodistal de diente inferior, el cálculo de los demás dientes de la misma arcada a partir de un elemento dentario inferior se realiza de la misma forma que en el caso antes mencionado, y para el cálculo de los dientes superiores se realiza comparando el radio Bonwill y el radio cuerda de Carrea ${ }^{6}$. En ambos casos, la salida del sistema es la determinación de los diámetros mesiodistales y la métrica facial, basada en la relaciones antropométricas de Carrea ${ }^{6}$ (Tabla 2), que toma como elemento de cálculo el radio cuerda.

El esquema de la Figura 1 muestra el flujo de cálculo en el cual se basa el algoritmo. En el Anexo se incluye el algoritmo de cálculo y la Tabla 1 muestra los porcentajes de los diámetros mesiodistales en función de la hemiarcada.

Las ecuaciones que describen el modelo matemático se extrajeron del trabajo original de Bloise ${ }^{1}$, siendo que la relación entre radio cuerda y radio Bonwill para el cálculo de las dimensiones dentarias a partir de un elemento conocido superior fue modificada con el intuito de que el modelo fuera robusto y consistente en relación al cálculo de los valores medios denotados en la tabla de valores medios de Black ${ }^{1}$. El ajuste realizado para la relación entre el radio cuerda y el radio Bonwill ${ }^{1}$ es el siguiente:

$$
R_{C}=\frac{R_{B}}{1324}(1)
$$

donde $R_{C}$ representa el radio cuerda $(\mathrm{mm})$ y $R_{B}$ el radio Bonwill (mm).

El ajuste anterior realizado al modelo de cálculo propuesto originalmente por Bloise ${ }^{1}$ es necesario con el

Tabla 1. Porcentaje de diámetros mesiodistales del segmento anterior en función de la hemiarcada, reportado por Bloise ${ }^{1}$.

\begin{tabular}{rlcc}
\hline $\mathbf{i}$ & Elemento dentario & TBi: \% en función hemiarcada & Sai: \% segmento anterior \\
\hline 1 & Incisivo central superior & 13,740 & 39,130 \\
2 & Incisivo lateral superior & 9,770 & 27,826 \\
3 & Canino superior & 11,603 & 33,043 \\
4 & Primer premolar superior & 10,992 & \\
5 & Segundo premolar superior & 10,381 & \\
6 & Primer molar superior & 16,335 & 29,669 \\
7 & Segundo molar superior & 14,045 & 32,417 \\
8 & Tercer molar superior & 13,129 & 37,911 \\
9 & Incisivo central inferior & 8,333 & \\
10 & Incisivo lateral inferior & 9,104 & \\
11 & Canino inferior & 10,648 & \\
12 & Primer premolar inferior & 10,648 & \\
13 & Segundo premolar inferior & 10,956 & \\
14 & Primer molar inferior & 17,283 & \\
15 & Segundo molar inferior & 16,512 & \\
16 & Tercer molar inferior & 16,512 & \\
\hline
\end{tabular}




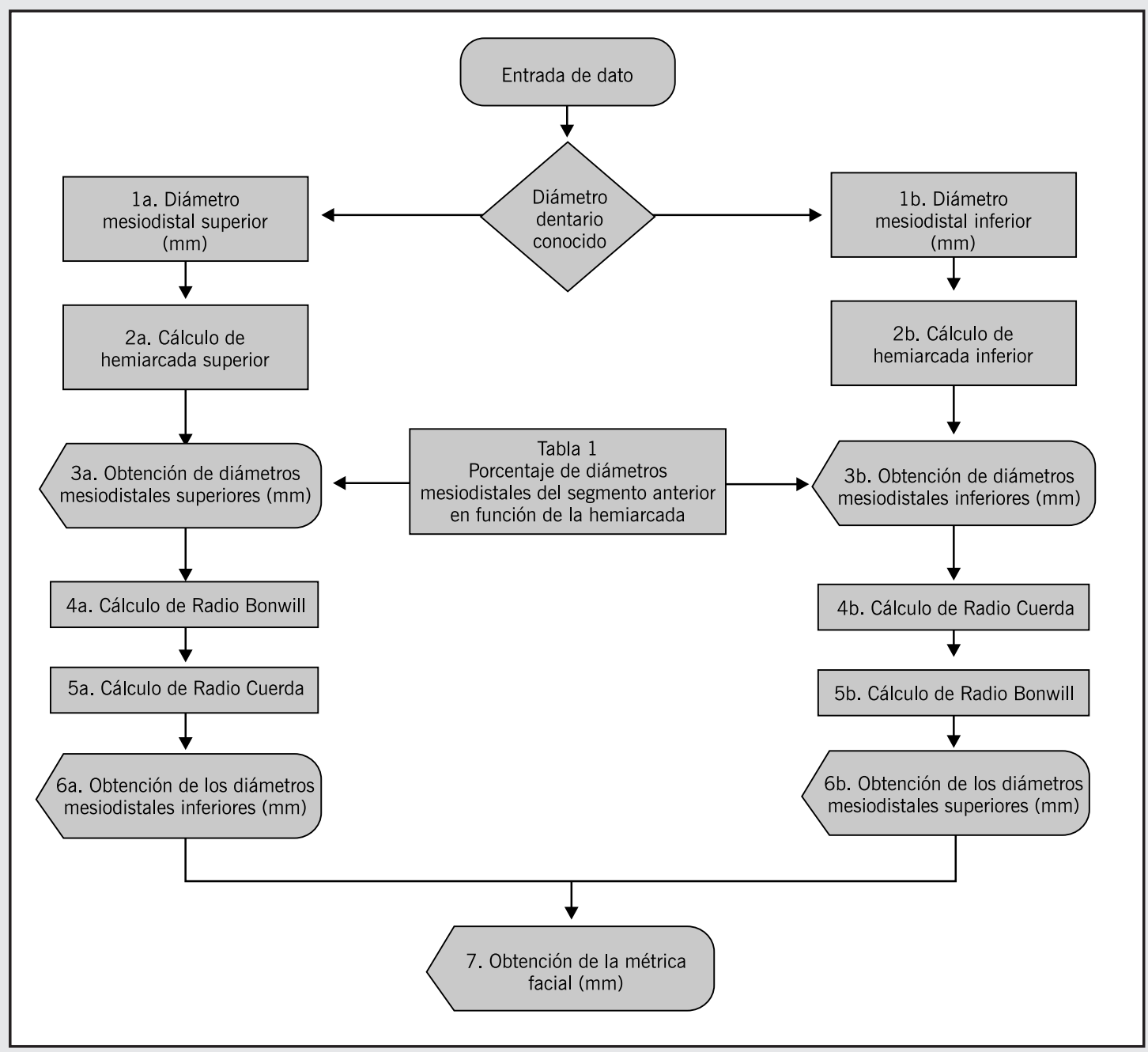

Figura 1.

Esquema del algoritmo de cálculo. fin de que los valores de entrada y salida del modelo verifiquen los valores dentarios medios sugeridos por Black ${ }^{1}$. Se trata de un ajuste matemático y no de un ajuste empírico, pero absolutamente necesario para que el modelo sea consistente para calcular los valores de los diámetros mesiodistales de la arcada superior cuando es conocido un elemento dentario inferior.

\section{Muestra experimental}

Se realizaron 106 medidas dentarias, provenientes de cráneos y modelos de yeso, todos seleccionados de la Cátedra de Odontología Legal y Forense. El número total de la muestra fue de 106 medidas dentarias y faciales equivalentes. Las piezas dentarias se midieron en su diámetro mesiodistal coronario, no se tuvieron en cuenta los diámetros vestibulolinguales ni la longitud radicular. Las medidas faciales fueron determinadas según la convención adoptada en el método de Bloise ${ }^{1}$. La Figura 2 muestra fotos de las mediciones realizadas.

Como puede verse en la Figura 2a, se cuenta con tres elementos dentarios de la mandíbula inferior. En este caso, se selecciona como elemento para el cálculo el primer molar inferior derecho, y las otras unidades dentarias como referencia para comparar el valor predictivo por el cálculo del método de Bloise ${ }^{1}$ con el valor medido experimentalmente. Esta situación es clasificada como caso 1 de acuerdo con el método de 
Tabla 2.

Relaciones en función del radio cuerda $(R)$, reportado por Bloise ${ }^{1}$. Métrica facial.

\begin{tabular}{|c|c|}
\hline Elemento conocido & Valor \\
\hline Límite externo de la arcada maxilar al punto dentario de Bonwill (h) & $\mathrm{h}=R_{c}(\sqrt{ } 12)$ \\
\hline Ancho de arcada maxilar (yy) & yy $=R_{c}(2 \sqrt{ } 3)$ \\
\hline Largo de la arcada maxilar (C) & $\mathrm{C}=3 R_{\mathrm{C}}$ \\
\hline Altura del triángulo de Bonwill (C) & $\mathrm{C}=R_{c}(\sqrt{ } 27)$ \\
\hline Radio de la circunferencia que circunscribe al $\Delta$ de Bonwill (R) & $\mathrm{R}=R_{c}(6 / \sqrt{ } 3)$ \\
\hline Lado deltriángulo protésico de Gysi (h) & $\mathrm{C}=3 R_{\mathrm{C}}$ \\
\hline Arco incisivo-canino (IC) & $\mathrm{IC}=R_{C}(\pi / 3)$ \\
\hline Arco-bicanino (BC) & $\mathrm{BC}=R_{c}(2 \pi / 3)$ \\
\hline Cuerda bicanina (CC) & $\mathrm{CC}=R_{C}(\sqrt{3})$ \\
\hline Altura del triángulo mandibular externo $(\mathrm{C})$ & $\mathrm{C}=R_{\mathrm{C}}(\sqrt{ } 147 / 2)$ \\
\hline Radio-circunferencia (R) del triángulo mandibular externo & $\mathrm{R}=R_{c}(7 / \sqrt{ } 3)$ \\
\hline Distancia condilea-externa-dentaria de Bonwill (h) & $\mathrm{h}=R_{c}(\sqrt{ } 45)$ \\
\hline Distancia mentogeniana (h) y dento-goniana (h) & $\mathrm{h}=R_{c}(\sqrt{ } 10)$ \\
\hline Distancia mento-dentaria de Bonwill (DB Men) & $\mathrm{DB}$ Men $=2 R_{c}$ \\
\hline Altura del triángulo bigoniacoespinal (es S) & es $\mathrm{S}=R_{c}(3 \sqrt{ } 3)$ \\
\hline Altura del triángulo biormafron-dentaria $(\mathrm{C})$ & $\mathrm{C}=R_{c}(\sqrt{3})$ \\
\hline Altura del triángulo biormafron mentoniano (N Men) & $\mathrm{N}$ Men $=R_{c}(\sqrt{ } 40)$ \\
\hline
\end{tabular}

Figura 2.

Figura 2a. Caso 1:

mandíbula inferior

con tres piezas dentarias

inferiores, 1er premolar

izquierdo, $2^{\circ}$ premolar

derecho y ler molar derecho.

Figura 2b. Caso 2: cráneo con un único diente superior.
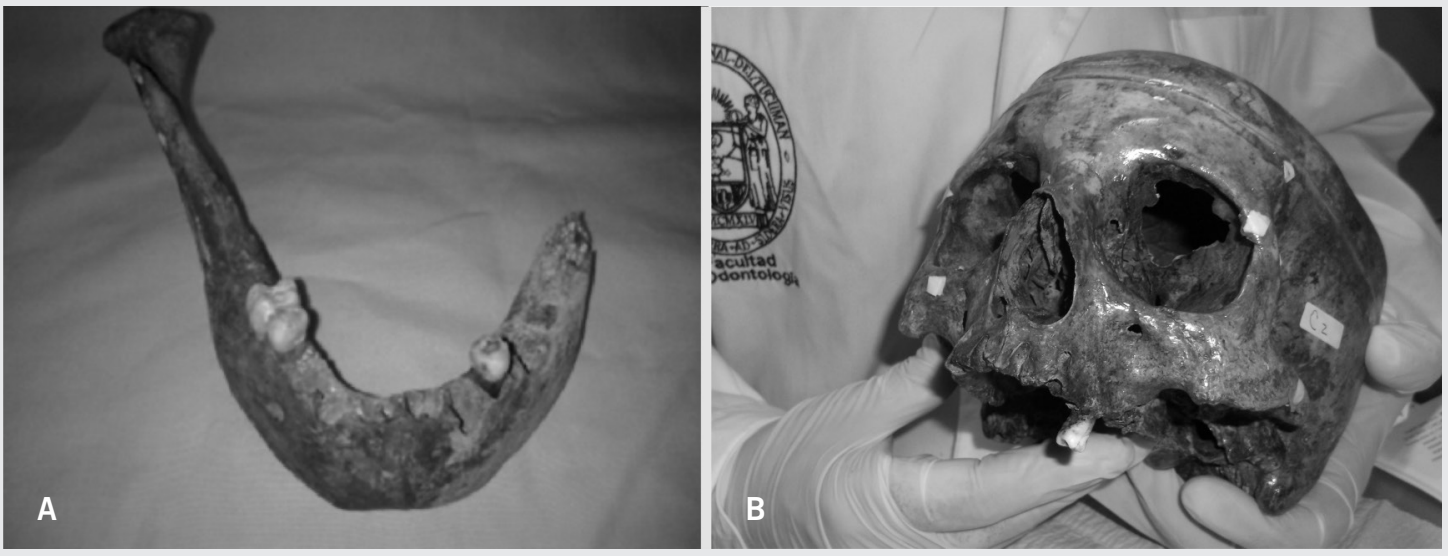

Bloise ${ }^{1}$. La Figura 2b representa un cráneo con una única pieza dentaria, siendo esta muestra la elegida para aplicar el método de Bloise ${ }^{1}$ a partir de un único diente superior, clasificada como caso 2.

\section{Materiales}

Para las mediciones se utilizaron un calibre digital, clase 2 , con precisión de 0,01 mm, y un calibre dentario con precisión de 0,01 mm. El segundo instrumento utilizado es más práctico que el primero desde el punto de vista de la práctica forense, una vez que permite realizar mediciones más fidedignas debido al diseño del instrumento, que termina en punta y posibilita alcanzar extremos entre unidades dentarias que otros instrumentos no lo consiguen (Figura 3). Todas las mediciones se realizaron portando guantes de látex profilácticos. 


\section{Protocolo de medición}

Las mediciones iniciales de la unidad dentaria seleccionada fueron realizadas diez veces por un mismo observador en diferentes instantes de tiempo. Luego se calcularon el valor medio y la desviación estándar. Para la medición de las dimensiones se adoptaron los criterios descritos por Bloise, sea para los diámetros mesiodistales. Las medidas de las unidades dentarias a comparar siguieron los mismos criterios de medición. La Figura 4 presenta la medición de cada uno de los casos antes mencionados.

\section{Procedimiento de cálculos}

Con el fin de validar los casos 1 y 2 descritos por Bloise como la determinación de las piezas dentarias a partir de una medición del diámetro mesiodistal de una unidad conocida, se desarrolló un algoritmo implementado en Excel $^{\circledR}$ (2010). El programa contempla las ecuaciones y las tablas de proporcionalidad descritas por Bloise en su trabajo original de $1953^{1}$, y la tabla de medias dentarias descrita por Black ${ }^{1}$.

El algoritmo de cálculo solicita inicialmente el diámetro mesiodistal de una unidad conocida, sea superior o inferior. En caso de un diente de la arcada superior, el algoritmo de cálculo determina los diámetros de las piezas dentarias de la arcada superior basándose en la tabla de diámetros mesiodistales medios descritos por Black ${ }^{1}$. Posteriormente calcula el valor del radio Bonwill ${ }^{1}$ y el radio cuerda de la arcada inferior. A partir del radio cuerda inferior se calculan los diámetros mesiodistales de las unidades inferiores. Habiéndose conseguido la magnitud del radio cuerda, en función de este se calculan las medidas craneofaciales (ver Tabla 2). El cálculo para el caso de una unidad de

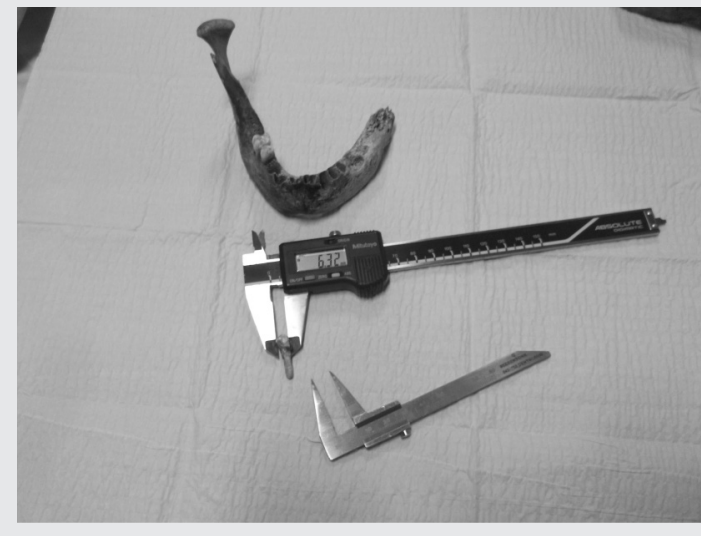

Figura 3.

Instrumentos utilizados en la adquisición de datos. En la parte superior de la imagen se observa una mandíbula con piezas dentarias; en la parte media, el calibre digital; $y$ en la parte inferior, el calibre dentario o de Vernier diámetro mesiodistal inferior conocida sigue un camino semejante, con la salvedad de que es necesario calcular un factor de corrección de la relación entre el radio cuerda y el radio Bonwill para ajustar que el modelo de cálculo verifique los valores medios de la tabla de valores medios de Black ${ }^{1}$.

\section{Análisis de los datos}

El segundo ajuste realizado al modelo de Bloise consistió en aplicar el método de Bland-Altman ${ }^{13-15}$ para comparar los datos experimentales con los datos calculados a partir del algoritmo. El análisis por el método Bland-Altman se realizó con un programa propio implementado en Matlab R2014 ${ }^{\circledR}$. En la secuencia se calculó un factor de corrección general a partir de los coeficientes de regresión lineal de Pearson ( $p<0,05)$. En este caso, el análisis de Pearson se realizó usando la herramienta estadística de Matlab
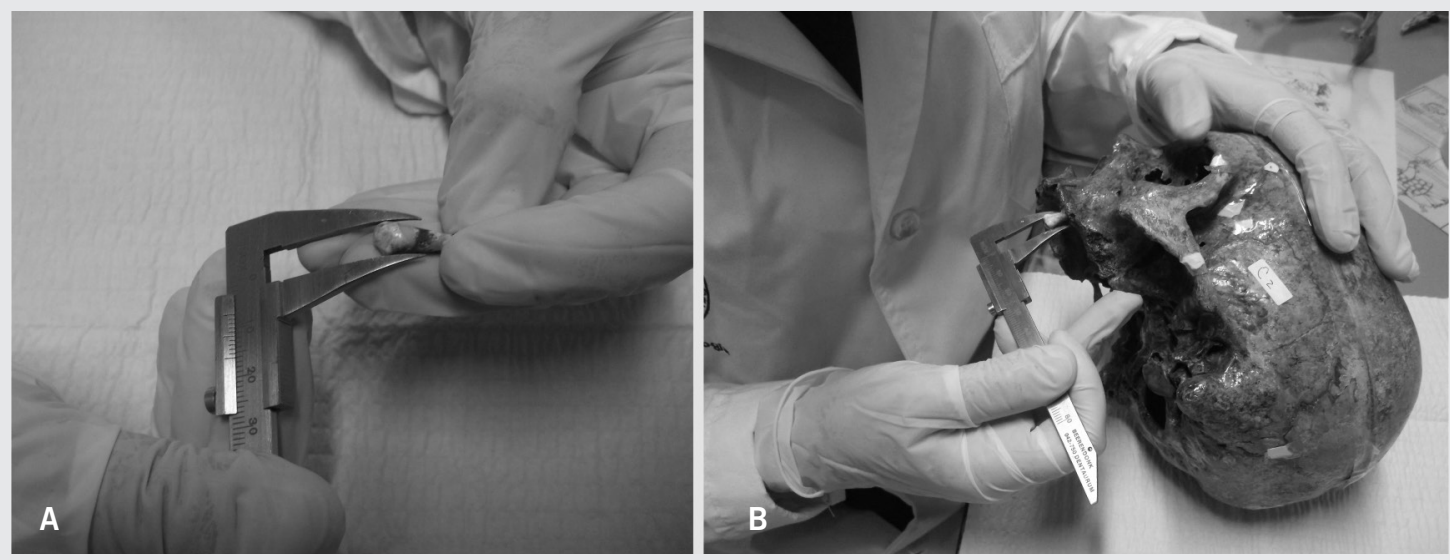

Figura 4.

Mediciones.

Figura 4a. $2^{\circ}$ premolar inferior.

Figura 4b. Medición de premolar superior izquierdo 
Figura 5.

Diagrama de Blant-Altman.

(a) Valores medidos vs. valores calculados por el

algoritmo sin factor de corrección.

(b) Valores medidos vs. valores corregidos de los diámetros mesiodistales

inferiores y superiores calculados con el método de Bloise corregido.
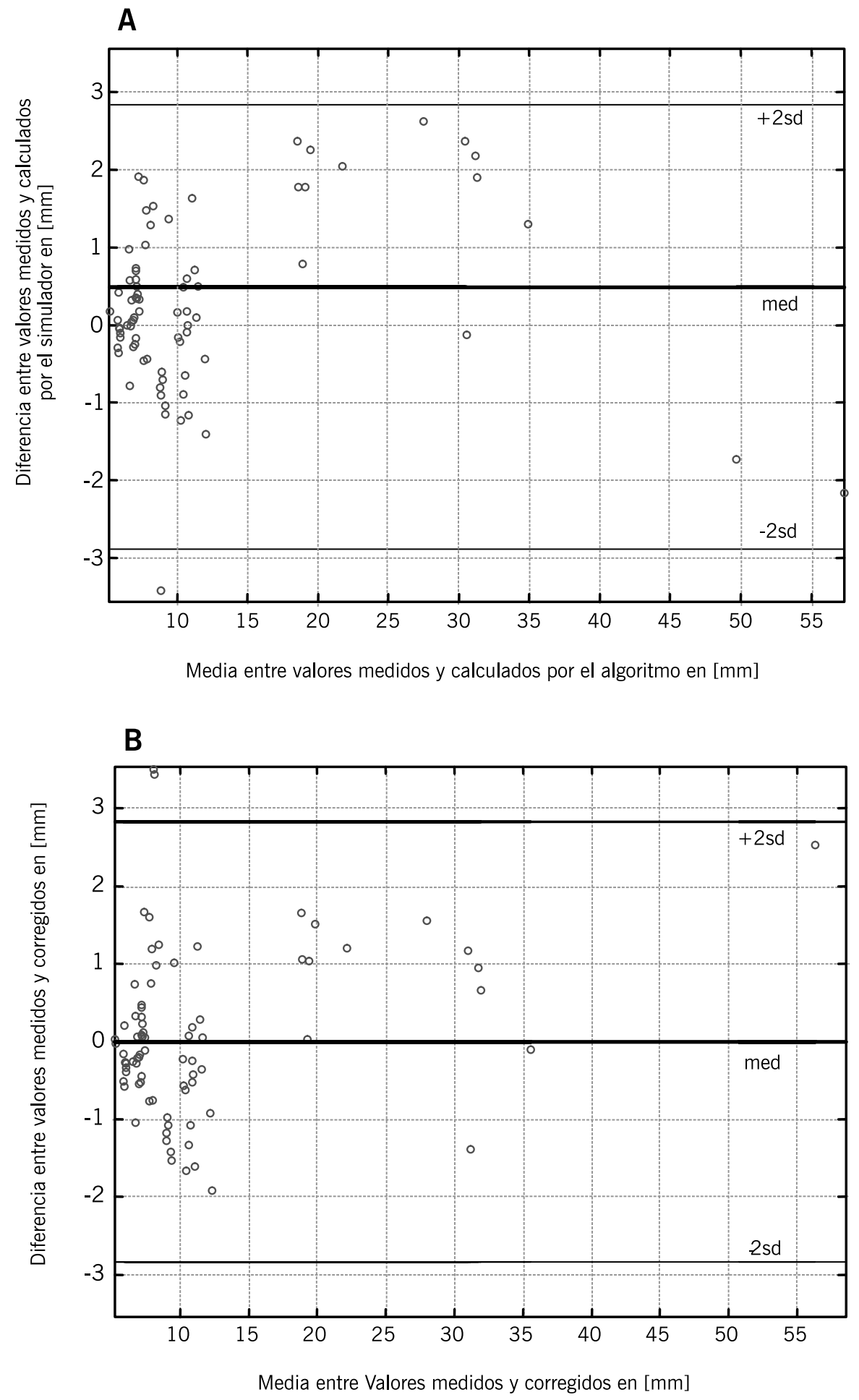
R2014 ${ }^{\circledR}$. Finalmente, se aplicó de nuevo el método de Bland-Altman ${ }^{13-15}$ entre los valores corregidos y los valores experimentales.

\section{Resultados}

Una vez realizada la verificación del modelo implementado a partir de los valores medios de Black se procedió al ajuste general del modelo antropométrico de Bloise para el caso de la determinación de las piezas dentarias a partir de una única medida de diámetro mesiodistal conocida.

La Figura 5a muestra el diagrama de Bland-Altman $^{13-15}$ para los valores medidos experimentalmente y los calculados por el algoritmo a partir de una pieza dentaria de diámetro conocido.

Se observa en la Figura 5 que la diferencia entre los valores medios y los calculados es mayor que cero, exactamente igual a $0,48 \mathrm{~mm}( \pm 1,41 \mathrm{~mm})$, sea tanto para piezas dentarias de calibre próximo a $55 \mathrm{~mm}$ como para aquellas de $10 \mathrm{~mm}$. Si bien la dispersión de las medidas se concentra entre los límites de $\pm 2 \mathrm{DE}$, la media es superior a cero, siendo DE la desviación estándar o típica (SD, Standard Deviation en idioma inglés).

Con el fin de ajustar las diferencias entre los valores medios medidos experimentalmente y calculados se determinó el coeficiente de Pearson $(r=0,991171$, $p<0,001$ ). De esta forma, los valores calculados por el algoritmo fueron corregidos a partir de la siguiente relación:

$$
\text { Cor }=(\text { Cal }-0,0183) / 0,9601
$$

donde Cal representa el valor calculado por el algoritmo (en $\mathrm{mm}$ ) y Cor es el nuevo valor corregido (en $\mathrm{mm}$ ).

La Figura 5b muestra el diagrama de Blant-Altman para los valores medidos experimentalmente y los valores corregidos por el algoritmo obtenidos por el método de Bloise ${ }^{1}$.

En la Figura 5b se observa que el valor medio de las diferencias (línea azul) es ahora igual a -0,0005 $\mathrm{mm}( \pm 1,41)$, valor muy próximo a cero y con la mismo DE que los datos anteriores y el mismo coeficiente de correlación de Pearson $(r=0,991$, $p<0,001)$. Es decir, a partir de los factores de corrección sugeridos en el ajuste de la ecuación 1 y el ajuste de la ecuación 2, los valores calculados por el algoritmo, para este caso particular de Bloise, se ajustan dentro de los límites admisibles para el valor experimental, siendo que el error máximo para la determinación de todos los elementos dentarios a partir de una única pieza dentaria conocida está en torno a $\pm 0,08 \mathrm{~mm}$, lo cual corresponde al $95 \%$ de los casos estudiados.

\section{Discusión}

A la luz de estos hallazgos podemos decir que el método de Bloise ${ }^{1}$, para el caso en que se calculan las dimensiones de los elementos dentarios de la arcada superior e inferior a partir de un único elemento dentario conocido, fue ajustado con dos factores de corrección y, fundamentalmente, fue validado el algoritmo.

Los resultados obtenidos muestran que el algoritmo es una importante y rápida herramienta de cálculo para relacionar una única medida dentaria con la métrica facial, de acuerdo con lo preconizado por Carrea ${ }^{6} \mathrm{y}$ Black $^{12}$. Estas medidas antropométricas, en conjunto con la determinación del $\operatorname{sex}^{12}$ y la altura, pueden ser determinantes para la identificación de un individuo a partir de restos fragmentados 3 .

La metodología presentada en este artículo fue implementada basándose en los trabajos de Badal ${ }^{12}$, se ha utilizado el método de Bland-Altman ${ }^{13-15}$ y se han obtenido los valores máximo y mínimo de las diferencias del conjunto de medidas, diámetros mesiodistales de un elemento conocido y calculado por el algoritmo, para establecer el grado de reproducibilidad y concordancia. La ventaja de utilizar este método es que permite visualizar rápidamente si las diferencias entre las medidas calculadas por el algoritmo y las medidas experimentales de referencia tienen una significación importante. De hecho, al aplicarlo en los datos medidos experimentalmente y en los datos calculados sin corrección (Figura 5a) permitió ajustar el modelo de cálculo con los coeficientes denotados en la ecuación 2 (Figura 5b). Este último procedimiento de ajuste tiene como antecedente la corrección realizada al método de Carrea por diferentes autores ${ }^{10,16,17}$.

La facilidad de contar con un algoritmo validado y corregido puede ser útil en el campo de la ortodoncia y tener amplia aplicabilidad para el dimensionamiento de prótesis, economizando tiempo y material. Cuando los diámetros mesiodistales de las piezas dentales artificiales pueden calcularse a partir de las dimensiones faciales, es posible diseñar las piezas dentarias artificiales de acuerdo con las proporciones establecidas. Para ortodoncia, el cálculo de los diámetros mesiodistales de todos los dientes de ambas 


\section{ANEXO}

\section{Algoritmo de cálculo}

1. Ingreso de dato: diámetro mesiodistal de un diente conocido $\varphi_{\mathrm{l}}[\mathrm{mm}]$, donde:

$\mathrm{i}=1, \ldots, 8$ : diámetro mesiodistal de diente superior.

$\mathrm{i}=9, \ldots, 16$ : diámetro mesiodistal de diente inferior.

$\varphi_{1}$ : incisivo central superior.

$\varphi_{2}$ : incisivo lateral superior.

$\varphi_{3}$ : canino superior.

$\varphi_{4}$ : primer premolar superior.

$\varphi_{5}$ : segundo premolar superior.

$\varphi_{6}$ : primer molar superior.

$\varphi_{7}$ : segundo molar superior.

$\varphi_{8}$ : tercer molar superior.

$\varphi_{9}$ : incisivo central inferior.

$\varphi_{10}$ : incisivo lateral inferior.

$\varphi_{11}$ : canino inferior.

$\varphi_{12}$ : primer premolar inferior.

$\varphi_{13}$ : segundo premolar inferior.

$\varphi_{14}$ : primer molar inferior.

$\varphi_{15}$ : segundo molar inferior

$\varphi_{16}$ : tercer molar inferior.

2. Cálculo de la hemiarcada de acuerdo con la de tabla de Bloise

Caso i $=1, \ldots, 8$

$$
H_{S}=100 * \varphi_{i} / T B_{i}
$$

donde $H_{s}$ : hemiarcada superior; $T B_{i}$; factor de la Tabla de Bloise

$$
\mathrm{i}=1, \ldots, 8 .
$$

Caso $\mathrm{i}=9, \ldots, 16$

$$
H_{1}=100 * \varphi_{i} / T B_{i}
$$

donde $H_{1}$ : hemiarcada inferior; $T B_{i}$; factor

de la Tabla de Bloise

$$
\mathrm{i}=9, \ldots, 16 .
$$

3. Cálculo de los diámetros mesiodistales de los elementos dentarios

Caso $\mathrm{i}=1, \ldots, 8$

$$
\begin{gathered}
\forall j \neq i \\
\varphi_{j}=H_{s} * T B_{j} / 100
\end{gathered}
$$

donde $H_{s}$ : Hemiarcada superior; $T B_{j}$ : factor de la Tabla de Bloise

$$
j \neq i, j=1, \ldots, 8 \text {. }
$$

Caso $\mathrm{i}=8, \ldots, 16$

$$
\begin{gathered}
\forall j \neq i \\
\varphi_{j}=H_{l} * T B_{j} / 100
\end{gathered}
$$

donde $H_{l}$ : Hemiarcada inferior; $T B_{j}$ : factor de la Tabla de Bloise

$$
j \neq i, j=9, \ldots, 16 .
$$

4. Cálculo del radio cuerda y del radio Bonwill

Caso i $=1, \ldots, 8$

$$
\begin{gathered}
R_{B}=\varphi_{1}+\varphi_{2}+\varphi_{3} \\
R_{C}=R_{B} / 1,324
\end{gathered}
$$

donde $R_{B}=$ radio Bonwill; $R_{C}=$ radio cuerda

$$
\begin{gathered}
\text { Caso } \mathrm{i}=9, \ldots, 16 \\
\qquad \begin{array}{c}
R_{C}=\left(\varphi_{9}+\varphi_{10}+\varphi_{11}\right) * 0,954 \\
R_{B}=R_{C} * 1,324
\end{array}
\end{gathered}
$$

5. Cálculo de los diámetros mesiodistales de los dientes inferiores a partir del diámetro mesiodistal de un diente superior conocido

$$
\varphi_{9}+R_{C} * S_{\alpha 9} / 100 / 0,954
$$

Cálculo de la hemiarcada inferior

$$
\begin{gathered}
H_{1}=\varphi_{9}{ }^{*} 100 / T B_{9} \\
\forall i=10, \ldots, 16 \\
\varphi_{i}=H_{1}{ }^{*} T B_{i} / 100
\end{gathered}
$$

6. Cálculo de los diámetros mesiodistales de los dientes superiores a partir del diámetro mesiodistal de un diente inferior conocido

$$
\varphi_{1}+R_{B} * S_{\alpha 1} / 100
$$

Cálculo de la hemiarcada superior

$$
\begin{gathered}
H_{1}=\varphi_{1} * 100 / T B_{1} \\
\forall i=2, \ldots, 8 \\
\varphi_{i}=H_{S} * T B_{i} / 100
\end{gathered}
$$

\section{Cálculo de las medidas craneofaciales}

Habiéndose conseguido la magnitud del radio cuerda, en función de este se calculan las medidas craneofaciales (ver Tabla 1). 
arcadas va a permitir saber con prioridad si, al hacer la corrección ortodóncica, esos mismos dientes colocados en su posición normal van a caber o no en el arco correspondiente ${ }^{1}$.

En el área forense tiene una aplicación muy destacada porque en numerosos casos se encuentran piezas dentarias aisladas y son una de las pruebas físicas más duraderas de la existencia de un individuo después de la muerte ${ }^{12}$. Los dientes se estudian en relación a su morfometría para establecer el sexo de la persona a través del índice de Aitchinson, que relaciona los diámetros mesiodistales de los incisivos centrales y laterales. El largo dentario y su relación con la distancia nasion-basion es indicativa de la raza mediante el índice de Flower ${ }^{18}$. El estudio para relacionar una pieza dentaria con las medidas faciales fue propuesto por Bloise, pero no hemos encontrado análisis que validen su modelo, el cual podría vincular una pieza dentaria con dimensiones craneofaciales y de esa manera reproducir el tejido óseo mediante programas computarizados para luego proceder a una reconstrucción facial de los tejidos blandos.

La metodología habitual para realizar reconstrucciones faciales debe contar con el cráneo y la mandíbula para aplicar técnicas bidimensionales o tridimensionales $^{18}$. La metodología propuesta y aplicada a partir del método de Bloise ${ }^{1}$ ajustado teniendo como punto de partida un diente permitiría relacionarlo con medidas craneofaciales, y con posterioridad podrían ampliarse los estudios referidos a las relaciones métricas craneofaciales iniciales de Bloise. Una de las medidas podría ser la relación entre el segmento dentario anterosuperior y el perímetro craneal ${ }^{19}$, debido a que estas relaciones no son consideradas por el método original.

Diferentes autores estudiaron o modificaron el método de Carrea para estimar la estatura humana a partir de piezas dentarias inferiores ${ }^{10,16}$, y otros autores utilizaron piezas dentarias superiores ${ }^{17}$, pero el método de Bloise ${ }^{1}$ no fue validado hasta el momento.

En este estudio inicial de Bloise sólo se consideraron los casos para determinar las dimensiones dentarias y la métrica facial a partir de una única pieza dentaria conocida. Los otros casos descritos por Bloise en su trabajo original ${ }^{1}$ no fueron considerados, pero son parte de un estudio que está siendo desarrollado y que será presentado en un artículo que es secuencia del presente.

\section{Conclusión}

A partir de la muestra estudiada en este trabajo queda demostrado que la medición del diámetro mesiodistal de una pieza dentaria es una metodología rápida y fácil de estudiar en contextos forenses para estimar el diámetro mesiodistal de todos los elementos y las medidas faciales, aplicando el método de Bloise ajustado en este trabajo.

\section{Contribuciones de los autores}

MAR condujo las mediciones para este estudio, desarrolló el algoritmo, analizó los datos y contribuyó en la escritura del manuscrito. DGG orientó en el trabajo a MAR y realizó la escritura del manuscrito.

\section{Agradecimientos}

DGG agradece los recursos financieros de AUGM/USP, Brasil, y de CIUNT, Tucumán, Argentina, en el Marco Institucional/Internacional del Grupo Montevideo para la realización de esta investigación conjunta. MAR agradece a la Prof. Mg. María I. Ferrari por facilitar la infraestructura de la Cátedra de Odontología Forense y Legal, Facultad de Odontología, UNT. MAR y DGG agradecen al Prof. Dr. Robson Rodrigues da Silva (NPT/UMC, Brasil) por la revisión del algoritmo descrito en este manuscrito.

Los autores declaran no tener ningún conflicto de intereses.

\section{Bibliografía}

1. Bloise V. La identificación humana por el sistema maxilo-mandíbulo-dentario. Buenos Aires: El Ateneo; 1953.
2. Morel P. La antropología física/Pierre Morel. Trad. por Sara Billino. $4^{a}$ ed. Buenos Aires: Editorial Universitaria; 1977. p. 7-59. 
3. Hillson S. Dental antropology. Londres: Cambridge University Press; 1996. p. 31-45.

4. Wasterlain SN. Males da boca. Estudio da patología oral numa amostra das Colecções Osteologicas Identificadas do Museu Antropológico da Universidade de Coimba (finais de séc XIX/ inícios de séc XX). Tesis doctoral. Universidade de Coimbra; 2006.

5. Carrea JU. Ensayos odontométricos. Tesis. Buenos Aires: Universidad Nacional de Buenos Aires; 1912.

6. Rodríguez JV. Introducción a la antropología dental. Cuadernos Antrop. 1989;19:1-41.

7. Bernabé E, Lagravére MO, Flórez C. Permanent dentition mesiodistal and bucco-lingual crown diameters in a Peruvian sample. Inter J Dental Anthropol. 2005;6:1-13.

8. Rodríguez JV. Dientes y diversidad humana: avances de la antropología dental. Santa Fe de Bogotá: Universidad Nacional de Colombia; 2003.

9. Briñón N. Odontología legal y práctica forense. Buenos Aires: Purinzon; 1984.

10. Cavalcanti AL, Porto DE, Maia AMA, Melo TRNB. Stature estimation by using the dental analysis: comparative study between Carrea's and the modified methods. Rev Odontol UNESP. 2007;36(4):335-9.

11. Gajardo P, Gajardo P, Torres S, Zavando D, Suazo GI. Determinación de la estatura a partir del arco y radiocuerda maxilar. Int J Odontostomat. 2011;5(3):267-9.

12. Badal J. Métodos odontométricos para la estimación del sexo en individuos adultos y subadultos. Tesis doctoral. Granada: Universidad de Granada; 2012.
13. Altman DG, Bland JM. Measurement in medicine: the analysis of method comparison studies. The Statistician. 1983;32:307-17.

14. Bland JM, Altman DG. Statistical methods for assessing agreement between two methods of clinical measurement. Lancet. 1986;327(8476):307-10.

15. Bland JM, Altman DG. Measuring agreement in method comparison studies. Statistical Methods in Medical Research. 1999;8(2):135-60.

16. Silva M. Estimativa da estatura do indivíduo com a utilização de um grupo de dentes da mandíbula. Rev Paul Odontol. 1990;12(4):18-28.

17. Correia LN. Validação do índice de Carrea por meio de elementos dentais superiores para a estimativa de estatura humana. Tesis maestrando. Piracicaba: Faculdade de Odontologia de Piracicaba, Universidade Estadual de Campinas; 2011.

18. Merabishvilli G. Superposición de imágenes en identificación facial bidimensional. Tesis doctoral. Barcelona: Facultad de Medicina, Departamento de Salud Pública, Universidad de Barcelona; 2006.

19. Martínez Rubio C. Determinación de la relación del segmento dentario con el perímetro craneal en el área urbana de los departamentos de Zacapa, Izabal, El Progreso y Chiquimula. Región de salud III y consolidado. Aplicación en odontología restaurativa. Tesis doctoral. Guatemala: Facultad de Odontología, Universidad de San Carlos de Guatemala; 2003 